\title{
Prospects for Supersymmetry at the LHC \& Beyond
}

\author{
John Ellis*广 \\ Theoretical Particle Physics and Cosmology Group, Department of Physics, \\ King's College London, Strand, London WC2R 2LS, U.K; \\ Theory Division, Physics Department, CERN, CH 1211 Geneva 23, Switzerland \\ E-mail: John.Ellis@cern.ch \\ KCL-PH-TH/2015-48, LCTS/2015-36, CERN-PH-TH/2015-250
}

\begin{abstract}
Run 1 of the LHC has provided three new motivations for supersymmetry: the need to stabilize the electroweak vacuum, the mass of the Higgs boson, and the fact that its couplings are Standard Model-like (so far). The prospects for discovering (and measuring) supersymmetry during future runs of the LHC are discussed in the frameworks of the constrained MSSM (CMSSM), models with non-universal soft supersymmetry-breaking contributions to Higgs masses (NUHM1,2) and the phenomenological MSSM with 10 arbitrary soft supersymmetry-breaking parameters (pMSSM10). In addition to the classic searches for missing transverse energy, searches for longlived charged sparticles may also be promising. If supersymmetry does show up at the LHC, there are good prospects for measurements of the spectrum that can be compared with the indirect indications from other experiments. On the other hand, a higher-energy future circular proton-proton collider may be the best option for discovering supersymmetry if it does not appear at the LHC.
\end{abstract}

18th International Conference From the Planck Scale to the Electroweak Scale 25-29 May 2015

Ioannina, Greece

\footnotetext{
* Speaker.

$\dagger$ I thank members of the MasterCode Collaboration: http://mastercode. web.cern.ch/mastercode/ and other collaborators on work described here, which has been supported in part by the European Research Council via the Advanced Investigator Grant 267352 and by the UK STFC via the research grant ST/L000326/1.
} 


\section{Introduction}

For lovers of supersymmetry, the good news from Run 1 of the LHC has been that there has been no evidence for any rival scenario for physics beyond the Standard Model (SM). The hapless fate of Higgsless models has been sealed, and Run 1 of the LHC found no signs of extra dimensions or compositeness. On the other hand, the bad news has been that there has also been no direct evidence for superymmetry. Nevertheless, I argue that there have been three indirect pieces of evidence favouring supersymmetry: the measured masses of the Higgs boson and the top quark suggest that the electroweak vacuum would be unstable within the SM (whereas it could be stabilized by supersymmetry), the measured Higgs mass is within the range predicted by simple supersymmetric models, and these also predicted successfully that its couplings would be similar to those in the SM. Advocates of many alternatives should be more discouraged than we lovers of supersymmetry. We lovers of supersymmetry should redouble our ardour, renewing searches for missing transverse energy (MET) and looking for possible long-lived charged sparticles. This talks presents my personal take on the remaining prospects for discovering (and measuring) supersymmetry at Run 2 and future runs of of the LHC, as well as previewing the prospects for future circular colliders.

\section{The Collapse of the Electroweak Vacuum, and How to Avert it}

In the SM, the quartic Higgs self-coupling $\lambda$ is renormalized by itself and by its Yukawa coupling to the top quark. The latter renormalization is negative, tending to drive $\lambda<0$ and induce vacuum instability at a scale $\Lambda_{I}$, which was estimated in [1] to be

$$
\log _{10}\left(\frac{\Lambda_{I}}{\mathrm{GeV}}\right)=11.3+1.0\left(\frac{m_{H}}{\mathrm{GeV}}-126\right)-1.2\left(\frac{m_{t}}{\mathrm{GeV}}-173.10\right)+0.4\left(\frac{\alpha_{s}\left(m_{Z}\right)-0.1184}{0.0007}\right) .
$$

Uisng the central values of the current world averages of $m_{t}=173.34 \pm 0.76 \mathrm{GeV}$ [2], $m_{H}=$ $125.09 \pm 0.24 \mathrm{GeV}$ [3] and $\alpha_{s}\left(M_{Z}\right)=0.1184 \pm 0.0006$ [4], it seems that the current electroweak vacuum is metastable, as seen in Fig. 1, and adding the uncertainties in quadrature in (2.1) one estimates

$$
\log _{10}\left(\frac{\Lambda_{I}}{\mathrm{GeV}}\right)=11.1 \pm 1.3 .
$$

Taken in isolation, the new CMS measurement of $m_{t}=172.44 \pm 0.49 \mathrm{GeV}$ [5] would modify this to $\log _{10}\left(\Lambda_{I} / \mathrm{GeV}\right)=11.6 \pm 0.7$. This would be changed to $\log _{10}\left(\Lambda_{I} / \mathrm{GeV}\right)=11.2 \pm 0.9$ if one also took $\alpha_{s}\left(M_{Z}\right)=0.1177 \pm 0.0013$ as might be suggested by a re-evaluation of lattice estimates [6]. If there were no physics beyond the SM, Higgs field values above $\Lambda_{I}$ would have a lower energy than the current SM vacuum, which is therefore unstable (though very long-lived).

But should one worry at all about this apparent instability? Certainly, we must hope for greater accuracy in the experimental measurements of $m_{t}$ and $\alpha_{s}\left(M_{Z}\right)$ to establish definitively whether we are in the region that would be meta/unstable within the SM. Also, since the lifetime for vacuum decay is probably much longer than the age of the Universe, one may wonder whether its metastability is a relevant issue. I would answer 'yes', for two reasons. 1) If our vacuum is not the true one, why is our present vacuum energy apparently adjusted to a very small in natural units? Moreover, calculations indicate that, if there is a lower-energy state out there, one would have expected 


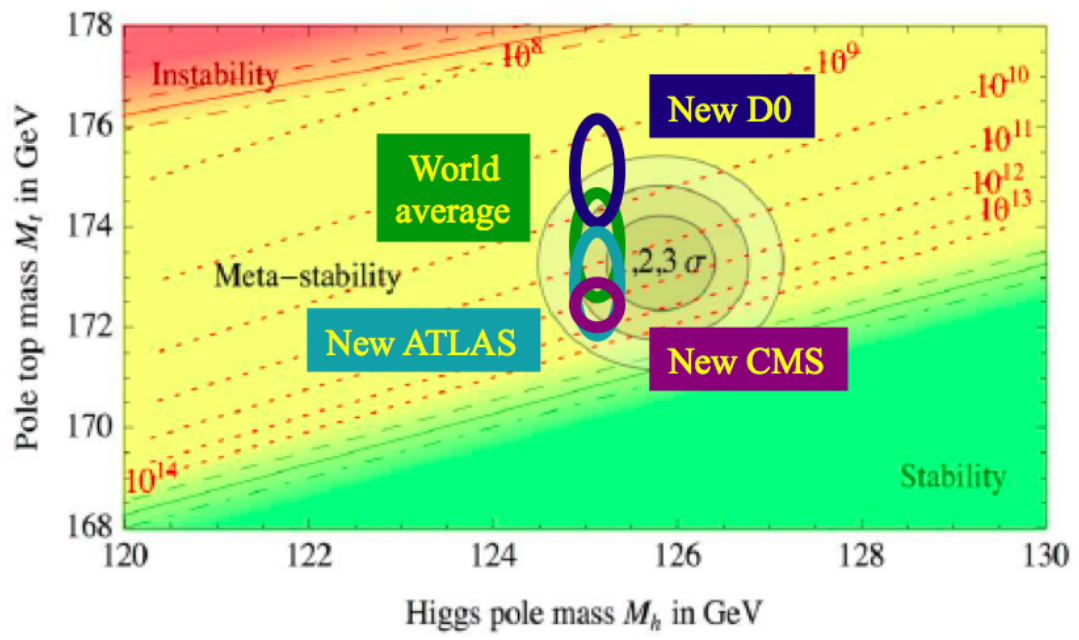

Figure 1: The $\left(m_{H}, m_{t}\right)$ plane showing regions of vacuum (meta/in)stability [1] and the 68\% CL regions favoured by LHC measurements of $m_{H}$ [3] and the 2014 world average measurement of $m_{t}$ [2], as well as more recent measurements by CMS, in particular [5].

most of the Universe to have fallen into it thanks to the large Higgs field fluctuations in the early Universe [7], as illustrated in Fig. 2. Averting this potential catastrophe requires some new physics beyond the SM, which could be supersymmetry [8], though many other types of new physics, such as dimension- 6 operators [9], could also do the job.

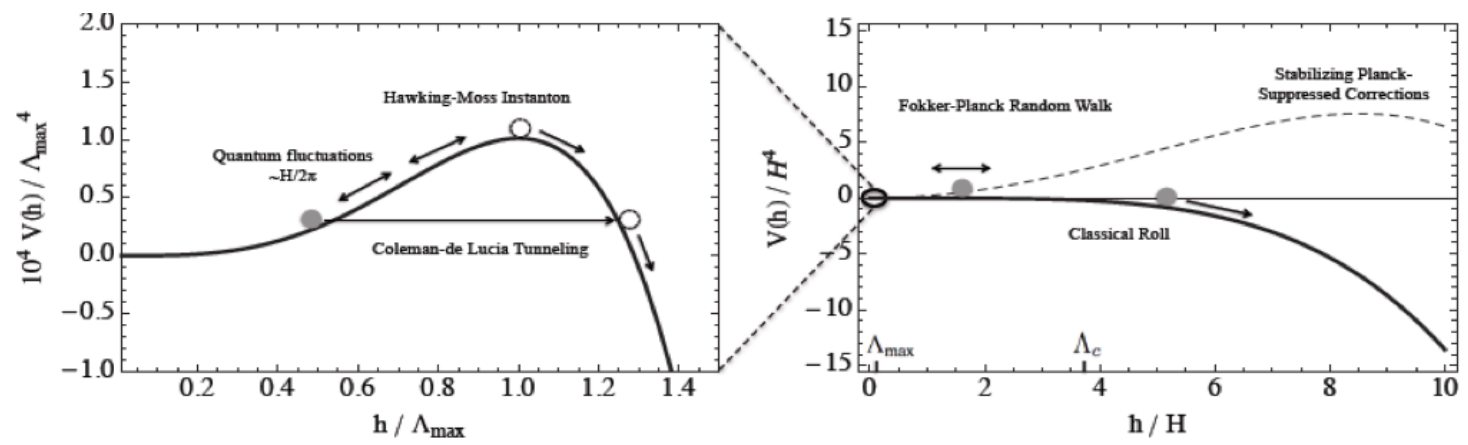

Figure 2: In the early Universe there would have been (left panel) an enhanced probability for a transition away from the neighbourhood of the electroweak vacuum towards large Higgs field values [7], and (right panel) averting this catastrophe would have required new physics such as supersymmetry [8] or higherdimensional interactions with coefficients suppressed by powers of the Planck mass [9].

\section{Floreat Supersymmetry, but which Model?}

As already mentioned, stabilizing the electroweak vacuum is just one of three new motivations for supersymmetry provided by data from LHC Run 1, the others being its successful predictions that $m_{h} \lesssim 130 \mathrm{GeV}$ [10] and that its couplings would be similar to those in the SM [11]. In contrast, whilst the Higgs mass and couplings could be accommodated within a generic composite model, this is not such an automatic prediction. These new motivations for supersymmetry can be added 
to all the traditional ones including the naturalness of the mass hierarchy, grand unification, its necessity within string theory and the fact that supersymmetric models contain a very plausible dark matter candidate [12].

Of course, it is disappointing that supersymmetric particles did not appear at the LHC during Run 1, and this certainly makes it a less complete solution to the naturalness problem. On the other hand, one's tolerance for a certain amount of fine-tuning is very much a personal matter, and supersymmetry at any scale is less unnatural than the SM without supersymmetry. In parallel, the LHC also did not produce any experimental evidence for any of the other proposed ways of addressing the naturalness issue and, while some new ideas are now emerging [13], none of them is yet as compelling as supersymmetry. Therefore, I am staying on the supersymmetric ship, even if it is leaking and listing a bit.

Most experimental searches for supersymmetry have been interpreted within the minimal supersymmetric extension of the SM (the MSSM), and I assume this framework in the following. This model conserves $R$ parity, and hence contains a suitable candidate for dark matter, which I also assume in the following. This leads to the characteristic missing-transverse-energy (MET) signature of supersymmetry that has been the basis for most LHC searches. Initially, these were often interpreted within the constrained MSSM (CMSSM) [14] in which the soft supersymmetrybreaking contributions to the scalar masses $m_{0}$, the gaugino masses $m_{1 / 2}$ and the trilinear scalar couplings $A_{0}$ were each assumed to be universal. However, this framework incorporates additional assumptions that may well not be valid. Natural flavour conservation would only impose universality on the masses of sfermions with the same quantum numbers, grand unification within SU(5) would allow different masses for sfermions in $\overline{\mathbf{5}}$ and $\mathbf{1 0}$ representations, and there is no obvious reason why the soft supersymmetry-breaking contributions to the masses of the two Higgs doublets should be the same as for the sleptons and squarks. Hence one may consider models with one or two non-universal Higgs mass parameters (NUHM1,2) [14, 15], and one may consider models in which no assumptions are made about the soft supersymmetry-breaking parameters, which are teated as purely phenomenological parameters (the pMSSM) [16].

\section{Global Fits}

In this Section I discuss some results from global fits to the CMSSM - which has 4 free parameters $m_{0}, m_{1 / 2}, A_{0}$ and $\tan \beta$, the NUHM1 - which also has an extra common Higgs mass parameter, the NUHM2 - which has two extra Higgs mass parameters $m_{1,2}$, and the pMSSM10 - a variant of the pMSSM with 10 free parameters, including 3 gaugino masses, different squark masses for the first/second and third generations, a common slepton mass, a common trilinear coupling $A_{0}$, $\mu, M_{A}$ and $\tan \beta$. For each model, we sample the multi-dimensional parameter space using the MultiNest algorithm with, e.g., $1.2 \times 10^{9}$ points to sample the pMSSM10 parameter space [19]. In addition to the Higgs mass, other LHC Higgs measurements and the negative results of LHC searches for supersymmetry, our global fits include many electroweak precision and flavour observables, $g_{\mu}-2$, the dark matter density and upper limits on spin-independent dark matter scattering. These measurements are used to construct a global $\chi^{2}$ function that we interpret using a frequentist approach [17, 18]. 
Fig. 3 displays $\left(m_{0}, m_{1 / 2}\right)$ planes for the CMSSM (upper left), the NUHM1 (upper right) and the NUHM2 (lower left), as well as the $\left(m_{\tilde{g}}, m_{\chi}\right)$ plane for the pMSSM10 (lower right panel). In each case, the best-fit point is indicated by a green star, and the $\Delta \chi^{2}=2.30$ and 5.99 contours (corresponding roughly to the 68 and 95\% CL boundaries) are indicated by red and blue lines, respectively. The $95 \% \mathrm{CL}$ region is shaded according to the mechanism that is most important for bringing the relic LSP density into the range of cold dark matter density favoured by Planck and other measurements, with the colour coding illustrated above the figure caption [19].
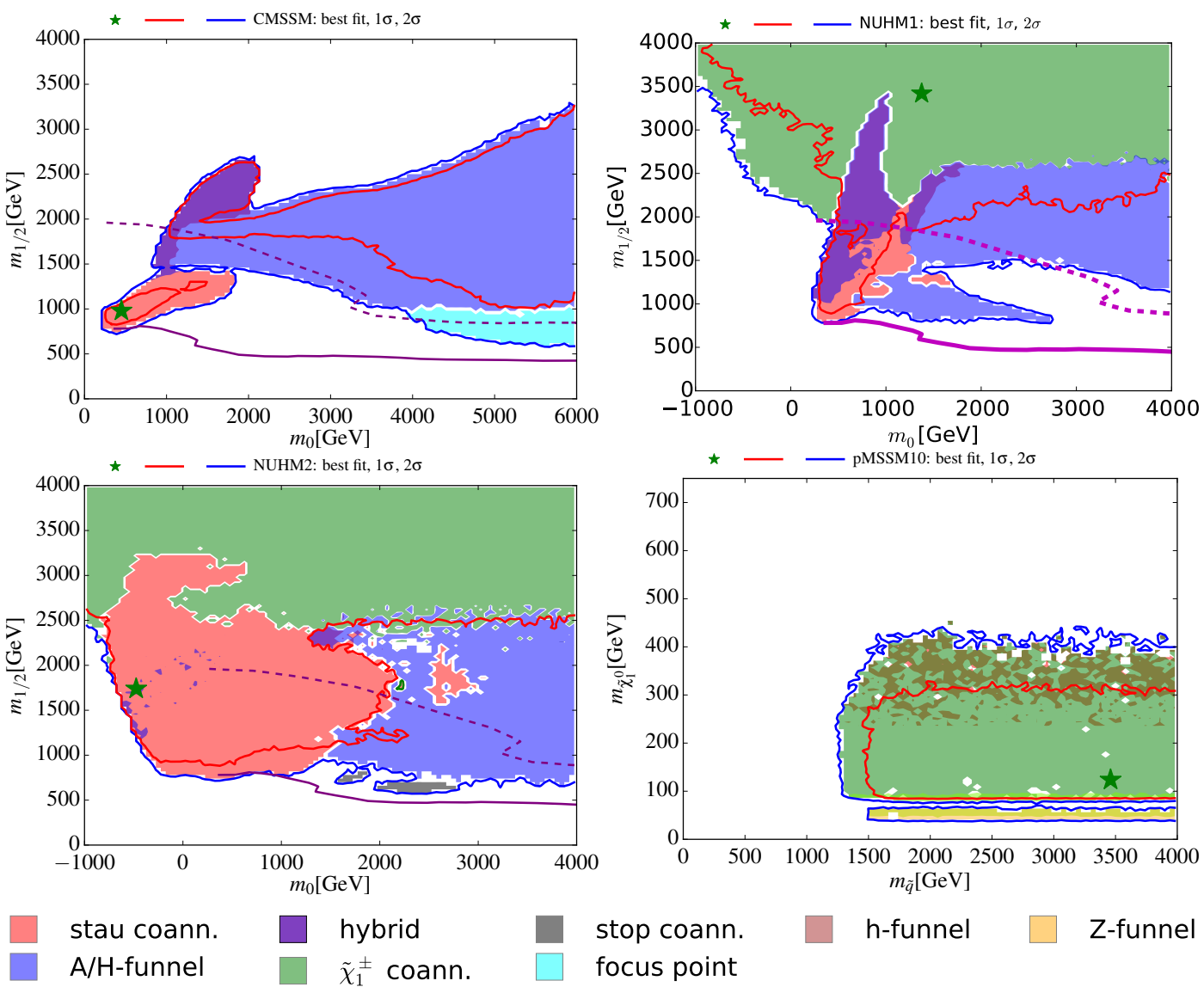

stop coann.

focus point

Figure 3: The $\left(m_{0}, m_{1 / 2}\right)$ planes in the CMSSM (upper left) [14], the NUHMI (upper right) [14] and the NUHM2 (lower left) [15], and the $\left(m_{\tilde{q}}, m_{\chi}\right)$ plane in the pMSSM10 (lower right panel) [16]. Regions in which different mechanisms bring the cold dark matter $(D M)$ density into the allowed range are shaded as described in the legend [19]. The red and blue contours are the $\Delta \chi^{2}=2.30$ and 5.99 contours found in global fits to these models, the green stars indicate the best-fit points, the solid purple contours show the current LHC 95\% exclusions from MET searches, and the dashed purple contours show the prospective 5- $\sigma$ discovery reaches for MET searches at the LHC with 3000/fb at $14 \mathrm{TeV}$, which also correspond approximately to the 95\% CL exclusion sensitivity with 300/fb at $14 \mathrm{TeV}$.

Fig. 4 [20] shows how the different observables contribute to building up the total $\chi^{2}$ function in the CMSSM at the global minimum (left column), along the $m_{0}$ axis (central column), and along the $m_{1 / 2}$ axis (right column). We see that the contribution of the flavour observables (dark grey) is almost independent of $m_{0}$ and $m_{1 / 2}$, as is the contribution of the electroweak precision observables (purple). On the other hand, the contribution from $g_{\mu}-2$ (teal) [21] is quite large at the 
minimum and increases with both $m_{0}$ and $m_{1 / 2}$. The contributions of the searches for the heavier supersymmetric Higgs bosons $H / A / H^{ \pm}$, and spin-independent dark matter scattering are never important, whereas the dark matter density constraint makes itself felt at large $m_{0}$ and $m_{1 / 2}$. On the other hand, the $b \rightarrow s \gamma$ constraint loses importance at large $m_{0}$ and $m_{1 / 2}$, whereas $B_{s} \rightarrow \mu^{+} \mu^{-}$[22] gains in importance. The $W$ mass makes some contribution at intermediate $m_{0}$ and $m_{1 / 2}$, whereas $m_{H}$ tends to disfavour both large and small $m_{0}$ and $m_{1 / 2}$. Finally the LHC jets + MET constraint disfavours low values of $m_{0}$ and $m_{1 / 2}$, and the global mimumum is largely determined by the tension between this constraint, $g_{\mu}-2$ and, to lesser extents, $m_{H}$ and $B_{s} \rightarrow \mu^{+} \mu^{-}$.
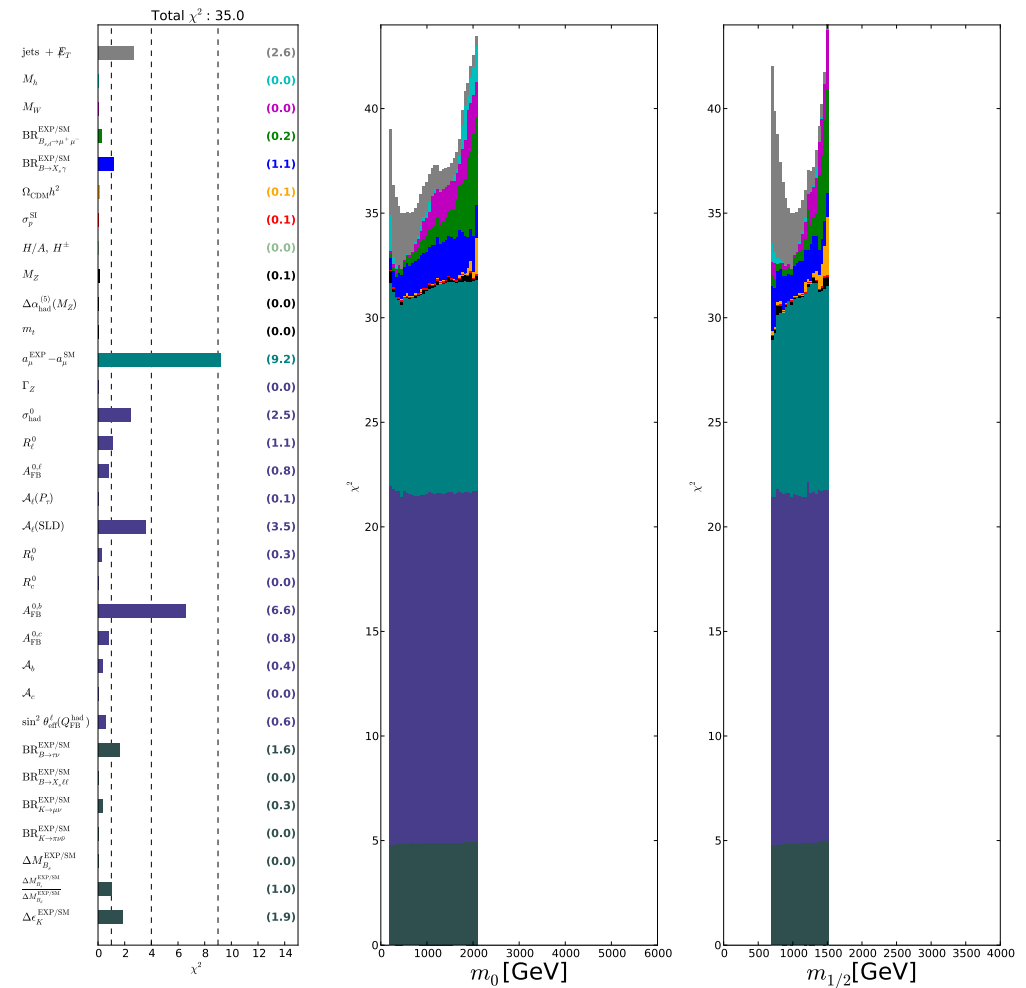

Figure 4: Results from a recent global fit to the CMSSM showing (left column) the contributions to the global likelihood at the best-fit point, and the marginalized likelihood as a function of (centre column) the $m_{0}$ mass and (right column) $m_{1 / 2}$ [20].

Fig. 3 also shows as solid (dashed) purple lines the present 95\% CL exclusion contour from searches for jets + MET events during Run 1 of the LHC (prospective $5-\sigma$ CL jets + MET discovery sensitivity with $3000 / \mathrm{fb}$ of data at $14 \mathrm{TeV}$ ) for the CMSSM, the NUHM1, the NUHM2 and the pMSSM10. We see that, except in the case of the NUHM2, the global 95\% CL contour generally lies significantly beyond the current jets + MET reach. This is due to the impacts of other constraints, notably the LHC measurement of the Higgs mass $m_{H}$, which, as was seen in Fig. 4, tends to favour relatively large sparticle masses.

On the positive side, we see in Fig. 3 that significant portions of the $68 \%$ CL regions in the CMSSM, the NUHM1 and the NUHM2 lie within the prospective reach of the LHC with 3000/fb of data at $14 \mathrm{TeV}$, including in particular substantial fractions of the regions where coannihilation with the stau is the dominant dark matter mechanism (shaded pink) [19]. In the case of the pMSSM10, 
this mechanism does not play such an important rôle: the most important dark mechanism is coannihilation with charginos $\chi^{ \pm}$(shaded green) [19]. However, also in this case a significant portion of the $68 \% \mathrm{CL}$ region lies within reach of future LHC searches.

\section{A Long-Lived Charged Sparticle?}

What would be the Run-2 signatures of supersymmetry in in these model scenarios? Clearly jets + MET will continue to be key, but other signatures may also be important. In particular, in the cases of the CMSSM, the NUHM1 and the NUHM2, there are substantial possibilities for detecting a long-live charged supersymmetric particle. In the pink-shaded regions of Fig. 3 the next-to-lightest supersymmetric particle (NLSP) is the lighter stau and the dark matter density is brought into the cosmological range by LSP-stau coannihilation. In this case the stau-LSP mass difference may be very small, as illustrated in the upper panels of Fig. 5 for the CMSSM (left panel) and the NUHM1 (right panel) [19]. The mass difference may be $1 \mathrm{GeV}$ or (much) less with a $\Delta \chi^{2}$ penalty $\lesssim 1$. This can lead to a relatively long lifetime for the $\tilde{\tau}_{1}$, as seen in the lower panels of Fig. 5, where the distributions in the $\left(m_{0}, m_{1 / 2}\right)$ planes of the CMSSM (left panel) and the NUHM1 (right panel) of model parameter sets with $\tilde{\tau}_{1}$ lifetimes in the range $\left[10^{-7}, 10^{3}\right] \mathrm{s}$ are shown ${ }^{1}$. As in Fig. 3, the red and blue contours delineate the $\delta \chi^{2}=2.30$ and 5.99 regions of these long-lived stau samples.

Charged sparticles with lifetimes $\gtrsim 10^{-7} \mathrm{~s}$ are likely to exit an LHC detector before decaying, providing metastable charged particle signatures such as a non-relativistic time of flight and anomalously high ionization. On the other hand, if the stau decays inside the detector, it may provide a disappearing-track signature. Supersymmetric final states at the LHC originate mainly from cascade decays of heavier sparticles. Therefore they may contain zero, one or two long-lived staus, and hence produce combinations of these signatures and also jets + MET [23]. A study has shown that the full extent of the stau coannihilation strip of the CMSSM can be explored by searching for these signatures at Run 2 of the LHC [24]. So far, the LHC experiments have analyzed their searches for long-lived sparticles mainly in the framework of near-degeneracy between the lightest chargino and neutralino: it is to be hoped that in the future these analyses will be extended to the case of a long-lived stau.

\section{Global Analysis of the pMSSM10}

In contrast to the CMSSM, the NUHM1 and the NUHM2, in the pMSSM10 [16] the NLSP is usually the lighter chargino $\chi_{1}^{ \pm}$, but the mass difference between the LSP and the NLSP is not expected to be very small, and a long-lived charged sparticle is disfavoured. Many searches with the LHC at $8 \mathrm{TeV}$ bear upon the allowed regions of the pMSSM10 parameter space, which were analysed using the Fastlim/Atom [25] and Scorpion [26] codes. The left panel of Fig. 6 displays the $\left(m_{\chi_{1}^{ \pm}}, m_{\chi}\right)$ plane in the pMSSM10, with the $68 \%$ and $95 \% \mathrm{CL}$ regions outlined as usual in red and blue, respectively. The diagonal dashed black lines correspond to $\Delta m \equiv m_{\tilde{\chi}_{1}^{ \pm}}-m_{\chi}=$ $0, M_{Z}$ and $m_{H}$. The coloured shadings correspond to the dominant $\chi_{1}^{ \pm}$decay modes, and the orange,

\footnotetext{
${ }^{1}$ If the stau had a longer lifetime, its decays would modify the successful predictions of conventional Big-Bang nucleosynthesis.
} 

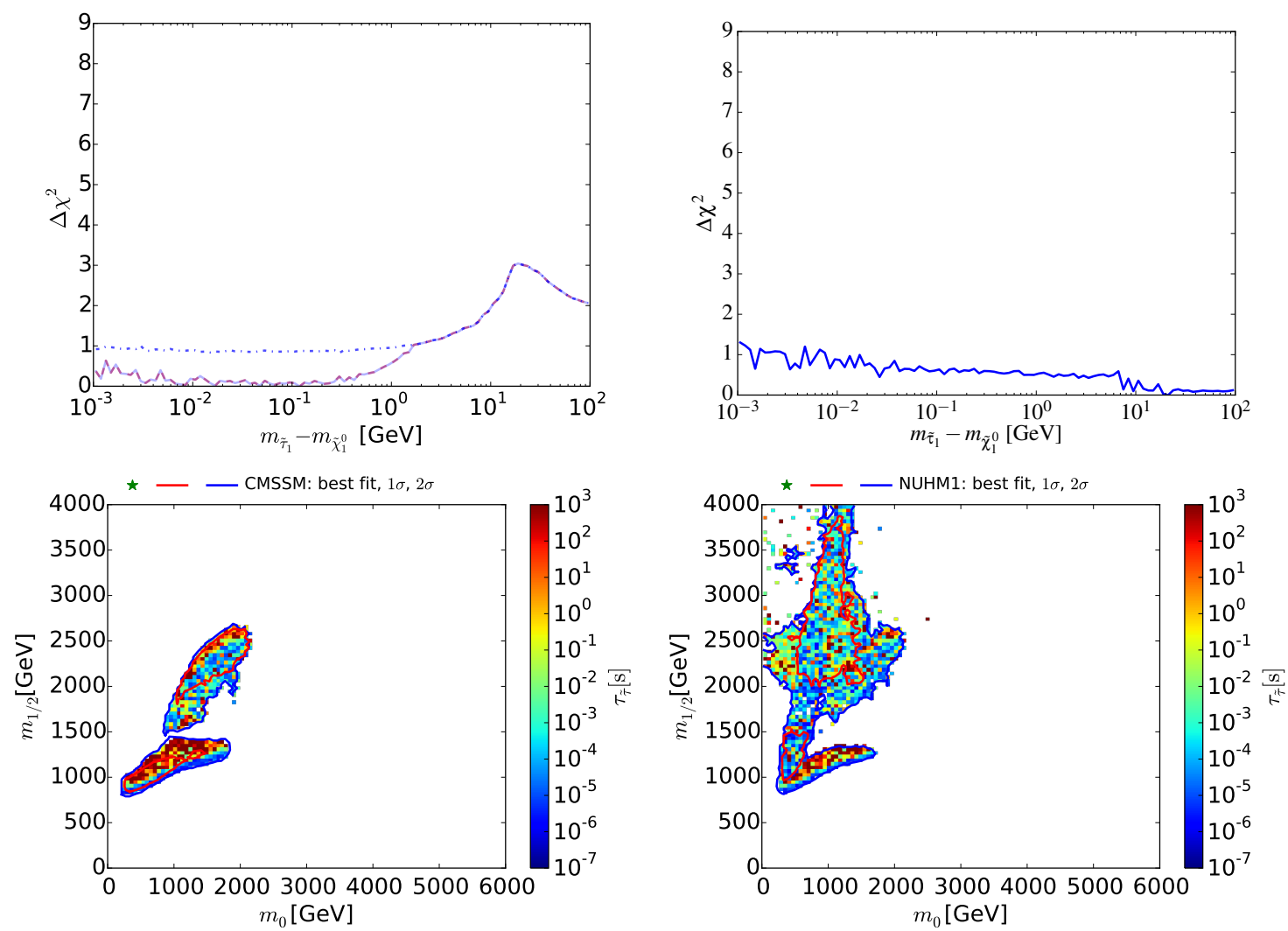

Figure 5: Upper panels: the one-dimensional $\Delta \chi^{2}$ profile likelihood functions for $m_{\tilde{\tau}_{1}}-m_{\chi}$ in the CMSSM (left) and the NUHM1 (right). Lower panels: the $\left(m_{0}, m_{1 / 2}\right)$ planes in the CMSSM (left) and the NUHM1 (right), with points colour-coded according to the $\tilde{\tau}_{1}$ lifetime found with the best-fit parameters for the corresponding values of $\left(m_{0}, m_{1 / 2}\right)$, and displaying only points with lifetimes $\in\left[10^{-7}, 10^{3}\right] s[19]$.

yellow and purple solid (dashed) lines represent the estimated reaches with 300 (3000)/fb of LHC data, assuming $100 \%$ branching ratios.

The pMSSM10 also offers the possibility of a relatively light stop squark, as seen in the right panel of Fig. 6. Here again, the many LHC searches that constrain the allowed regions of the pMSSM10 parameter space were analysed using the Fastlim/Atom [25] and Scorpion [26] codes. The diagonal dashed lines are where $\Delta m \equiv m_{\tilde{t}_{1}}-m_{\chi}=0, M_{W}+m_{b}$ and $m_{t}$. The light blue shading shows that $\tilde{t}_{1} \rightarrow b \tilde{\chi}_{1}^{ \pm}$is the dominant decay in much of the region displayed, and the sensitivity to this decay of the LHC with $300 / \mathrm{fb}$ is shown as a solid line in the corresponding colour (the reach with $3000 / \mathrm{fb}$ is similar). The solid black line shows the projected reach for $\tilde{t}_{1} \rightarrow t \chi$ if this is the dominant decay, which is however not the case in our pMSSM10 analysis.

Fig. 7 displays the breakdown of the global $\chi^{2}$ function in our analysis of the pMSSM10> We see that the Higgs production and decay data (olive green analysed using the Higgs Signals [27] code, notice the suppressed zero), the flavour observables (green) and the precision electroweak measurements (purple) make contributions that are rather insensitive to the values of the lighter smuon mass $m_{\tilde{\mu}_{R}}$ (centre column) and the LSP mass (right column). We also find relatively unimportant contributions from the dark matter constraints, $B_{s} \rightarrow \mu^{+} \mu^{-}$and the LHC searches. The 

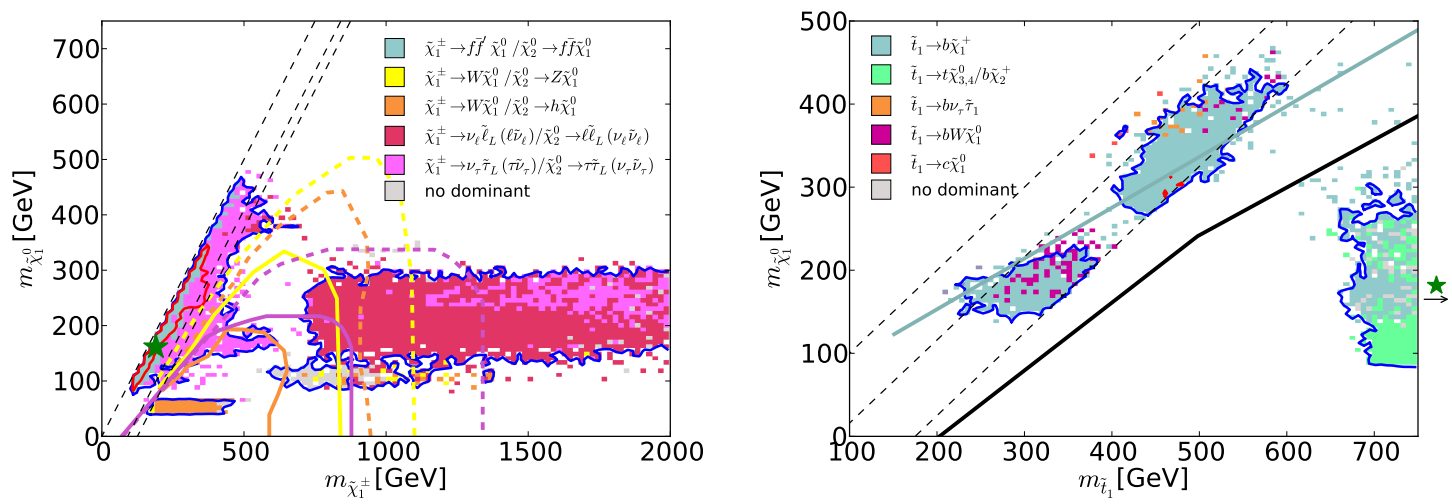

Figure 6: Upper left panel: The $\left(m_{\chi_{1}^{ \pm}}, m_{\chi}\right)$ plane with our 68 and $95 \%$ CL contours shown as solid red and blue lines, respectively. The coloured shadings indicate where the corresponding branching ratios exceed $50 \%$. Also shown as solid (dashed) yellow/orange/purple lines are the projected LHC $95 \% C L_{s}$ exclusion reaches for associated $\chi_{1}^{ \pm}$and $\chi_{2}$ production with decays via $W / Z / W / h / \tilde{\ell}_{L} / \tilde{v}_{\ell_{L}} / \tilde{\tau}_{L} / \tilde{v}_{\tau_{L}}$ with $300(3000)$ fb of data, if these decays are dominant. Upper right panel: The $\left(m_{\tilde{t}_{1}}, m_{\chi}\right)$ plane with our 68 and $95 \% C L$ contours shown as solid red and blue lines, respectively, as well as coloured regions where the indicated branching ratios exceed 50\%. The projected LHC sensitivity with 300/fb for $\tilde{t}_{1} \rightarrow \chi+t$ decays is shown as a thick black line, and the corresponding sensitivity for $\tilde{t}_{1} \rightarrow \chi_{1}^{ \pm} b$ decays (if they are dominant) is shown as a pale blue dashed line [16].

shape of the global $\chi^{2}$ function is mainly determined by $g_{\mu}-2$ (teal), which exerts a strong preference for small $m_{\tilde{\mu}_{R}}$ and also favours small $m_{\chi}$.

\section{Floreat $g_{\mu}-2$ ?}

This preference for small $m_{\tilde{\mu}_{R}}$ reflects the fact that, in contrast to the CMSSM and the NUHM1,2, the pMSSM10 can reconcile $g_{\mu}-2$ with the absence of supersymmetry at the LHC (so far) [16]. This contrast is also visible in Fig. 8, where we see that the pMSSM10 (solid black curve) can fit perfectly the experimental measurement of $g_{\mu}-2$ (solid red curve), whereas the CMSSM, the NUHM1 and the NUHM2 (blue solid, dashed and dotted lines, respectively) prefer values of $g_{\mu}-2$ that are close to the Standard Model value. In our analysis, this differs from the experimental measurement by $\sim 3 \sigma$. It is this inability to fit $g_{\mu}-2$ that is largely responsible for the poor overall quality of the the fits in the CMSSM, the NUHM1 and the NUHM2 (and also the SM). Naive estimates using the $\chi^{2}$ function indicate $p$-values below $10 \%$ for these models, and a similar estimate has been obtained in [17] using toys. On the other hand, we estimate a $p$-value $\sim 30 \%$ for the pMSSM10.

This comparison shows that elucidating the apparent discrepancy between the experimental measurement and the Standard Model calculation of $g_{\mu}-2$ is crucial for understanding the prospects for discovering supersymmetry in the near future. Is it possible that the theoretical calculation suffers from an unknown systematic uncertainty, possibly in the calculation of the light-bylight scattering contribution? Or is there some experimental effect that remains to be understood? If not, some new physics at the $\mathrm{TeV}$ scale is needed, and supersymmetry fits the bill, particularly 

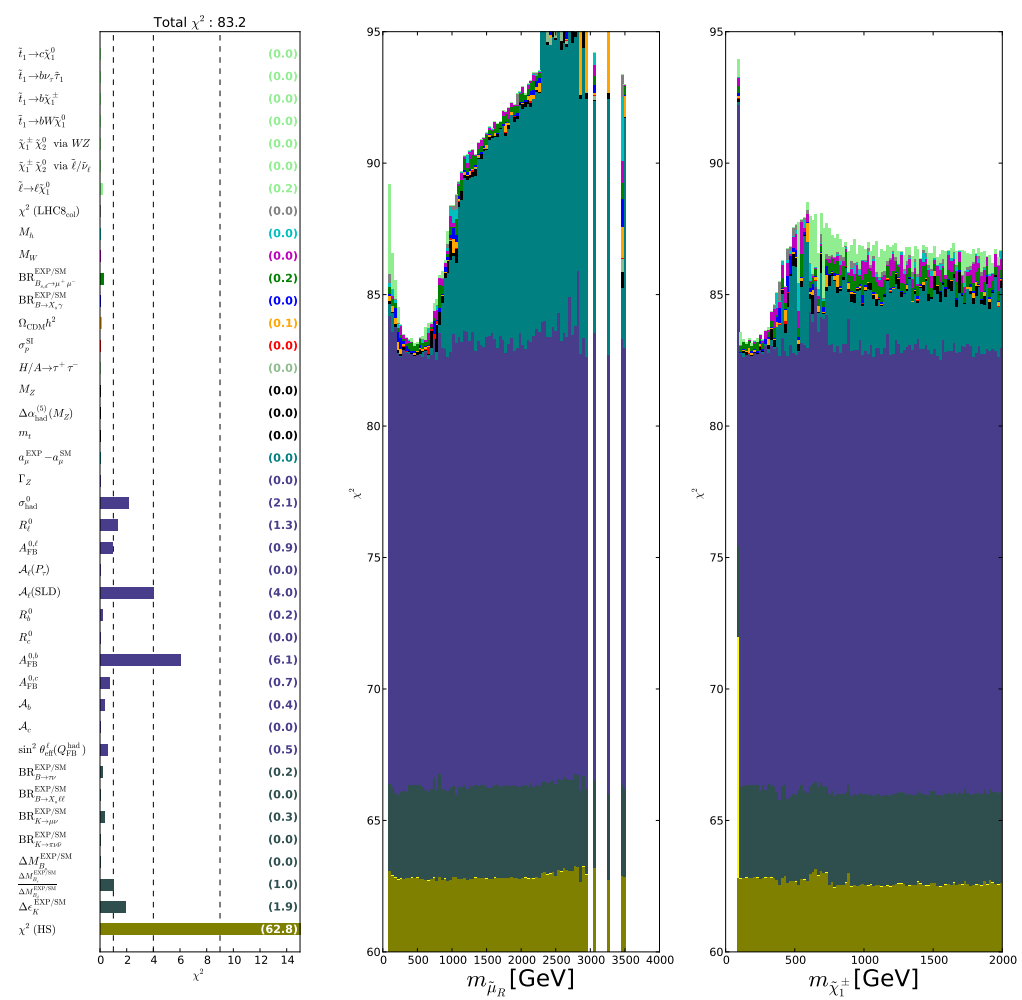

Figure 7: Results from a recent global fit to the pMSSM10 showing (left panel) the contributions to the global likelihood at the best-fit point, and the marginalized likelihood as a function of (centre panel) the $\tilde{\mu}_{R}$ mass and (right panel) the lighter chargino mass, $m_{\tilde{\chi}_{1}^{ \pm}}[20]$.

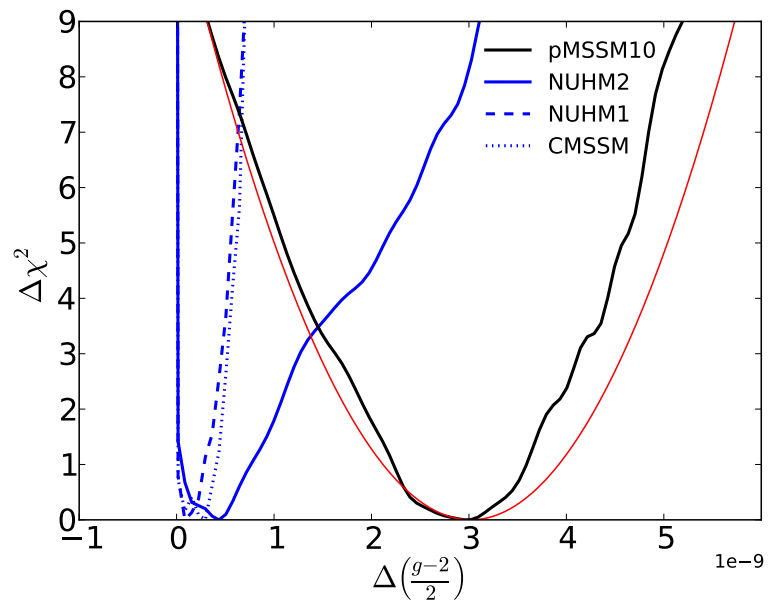

Figure 8: One-dimensional profile likelihoods for the supersymmetric contributions to $g_{\mu}-2$, from recent global fits to the CMSSM (blue dotted line), the NUHM1 (blue dashed line), the NUHM2 (blue solid line) and the pMSSM10 (black solid line), with the experimental likelihood (solid red line) shown for comparison [16].

within the pMSSM framework. The good news is that an experiment to remeasure $g_{\mu}-2$ with 
significantly improved accuracy is in an advanced stage of preparation at FNAL [28].

\section{Prospects for Discovering Supersymmetry at the LHC}

Fig. 9 displays the marginalised global $\chi^{2}$ functions for the masses of the gluino (left panel) and the squark (right panel) in the CMSSM (solid blue lines), the NUHM1 (dashed blue lines), the NUHM2 (dotted blue lines) and the pMSSM10 (solid black line) [16]. In the case of the gluino, we see that the pMSSM10 offers better prospects than the other models for a relatively light gluino with mass $\sim 1500 \mathrm{GeV}$, whereas the model predictions are more similar for the squark mass. Here the good news is that jets + MET searches at the LHC will become sensitive to $m_{\tilde{g}}, m_{\tilde{q}} \sim 3 \mathrm{TeV}$ with 3000/fb of integrated luminosity at $14 \mathrm{TeV}$, so there are decent prospects (but no guarantees) for discovering supersymmetry at the LHC in this channel.
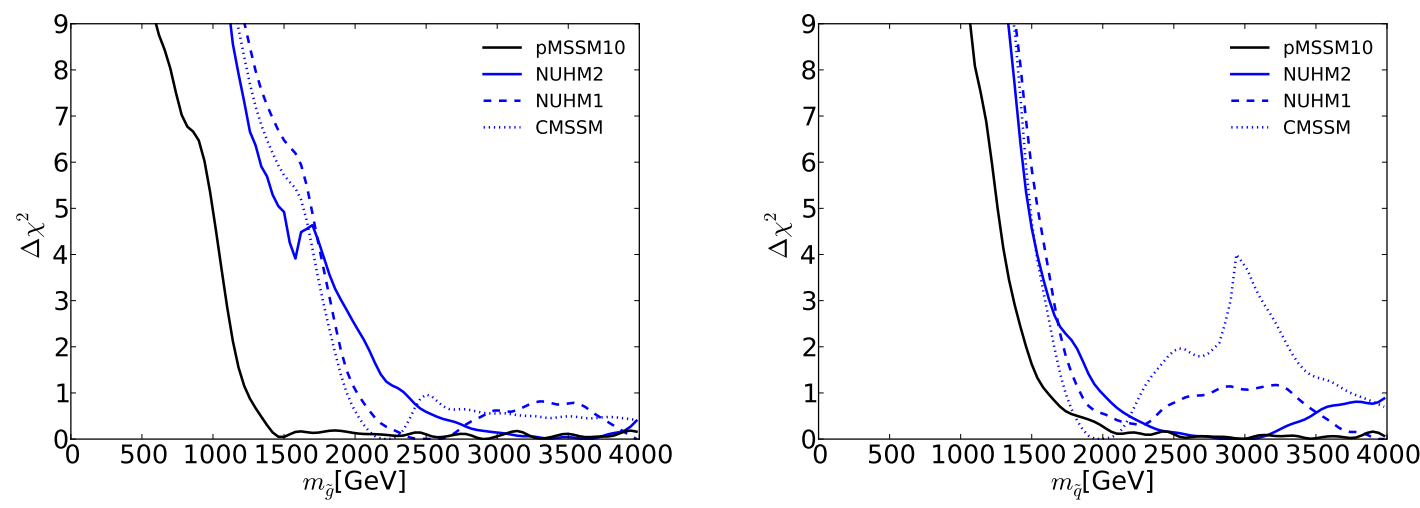

Figure 9: One-dimensional profile likelihood functions for $m_{\tilde{g}}$ and $m_{\tilde{q}}$ from recent global analyses of MSSM scenarios. In each panel the solid black line is for the pMSSM10, the solid blue line for the NUHM2, the dashed blue line for the NUHMI, and the dotted blue line for the CMSSM [16].

As we have seen previously, in the CMSSM, the NUHM1 and the NUHM2 there are also interesting prospects for discovering long-lived charged sparticles at the LHC, if the DM density is brought into the cosmological range by stau coannihilation. This is not likely in the pMSSM10, but in this model searches for charginos may be promising, as discussed in the previous Section. Table 1 [19] compiles the prospects for supersymmetry searches at the LHC in the CMSSM, the NUHM1, the NUHM2 and the pMSSM10, organised according to the dominant mechanisms for bringing the relic LSP density into the range allowed by cosmology. Also shown are the prospects for direct DM detection in each case. We see that in every instance where some mechanism may become dominant, there are prospects for detection either at the LHC or in direct DM search experiments (or both).

\section{Prospects for Measuring Supersymmetric Model Parameters}

Given the interesting prospects for discovering supersymmetric particles during future LHC runs, what are the prospects for measuring supersymmetric model parameters? The answer to this 


\begin{tabular}{|c||c||c|c|c|c|}
\hline \multicolumn{1}{|c||}{ DM } & \multicolumn{1}{c||}{ Exp’t } & \multicolumn{5}{c|}{ Models } \\
mechanism & & CMSSM & NUHM1 & NUHM2 & pMSSM10 \\
\hline$\tilde{\tau}_{1}$ & LHC & $\checkmark$ MET, $\checkmark$ LL & $(\checkmark$ MET, $\checkmark$ LL) & $(\checkmark$ MET, $\checkmark$ LL) & $(\checkmark$ MET), $\times$ LL \\
coann. & DM & $(\checkmark)$ & $(\checkmark)$ & $\times$ & $\times$ \\
\hline$\chi_{1}^{ \pm}$ & LHC & - & $\times$ & $\times$ & $(\checkmark$ MET $)$ \\
coann. & DM & - & $\checkmark$ & $\checkmark$ & $(\checkmark)$ \\
\hline$\tilde{t}_{1}$ & LHC & - & - & $\checkmark$ MET & - \\
coann. & DM & - & - & $\checkmark$ & - \\
\hline$A / H$ & LHC & $\checkmark A / H$ & $(\checkmark A / H)$ & $(\checkmark A / H)$ & - \\
funnel & DM & $\checkmark$ & $\checkmark$ & $(\checkmark)$ & - \\
\hline Focus & LHC & $(\checkmark$ MET $)$ & - & - & - \\
point & DM & $\checkmark$ & - & - & - \\
\hline$h, Z$ & LHC & - & - & - & $(\checkmark$ MET $)$ \\
funnels & DM & - & - & - & $(\checkmark)$ \\
\hline
\end{tabular}

Table 1: Compilation of assessments of the detectability of supersymmetry in the CMSSM, NUHM1, NUHM2 and pMSSM10 models at the LHC in searches for MET events, long-lived particles charged (LL) and heavy A/H Higgs bosons, and in direct DM search experiments, depending on the dominant mechanism for bringing the DM density into the cosmological range. The symbols $\checkmark,(\checkmark)$ and $\times$ indicate good prospects, interesting possibilities and unlikely prospects, respectively. The symbol - indicates that a DM mechanism is not important for the corresponding model [19].

question is highly model-dependent, and the experiments would surely do much better than we theorists can currently imagine.

Fig. 10 [29] shows some results from an exploratory study the simplest case of the CMSSM and the impact of the entry-level jet measurements of events containing squarks and gluinos. This analysis assumed that Nature has chosen the best-fit point found in the above-mentioned global analysis of the CMSSM, namely $m_{0}=670 \mathrm{GeV}, m_{1 / 2}=1040 \mathrm{GeV}, A_{0}=3440 \mathrm{GeV}$ and $\tan \beta=21$. Included in this analysis were prospective measurements of the total cross section for jet + MET events, the distribution in the MT2 variable, and the spectator jet energies in $\tilde{g} \rightarrow \tilde{q}_{R}+\bar{q}$ decay. Generally speaking, these measurements are more sensitive to the value of $m_{1 / 2}$ than to $m_{0}$, as seen in the left panel of Fig. 10 that assumes 300/fb of integrated luminosity at $14 \mathrm{TeV}$, and the right panel that assumes 3000/fb of integrated luminosity. The dashed red (blue) lines bound the 68 (95)\% CL regions found in the previous global analysis, and the solid red (blue) lines show the result on combining the prospective LHC measurements with this global analysis. We see that measurements with 300/fb would already restrict quite strongly the preferred region of parameter space, and that the effects of measurements with $3000 / \mathrm{fb}$ would be extremely restrictive.

This analysis shows that the LHC has good prospects for confronting indirect estimates of supersymmetric model parameters with more direct measurements. A successful confrontation, as shown in the CMSSM case in Fig. 10, would provide a non-trivial verification of supersymmetry at the quantum level. This could parallel the previous tests of the SM at the quantum level that led to successful predictions of the top and Higgs masses. 

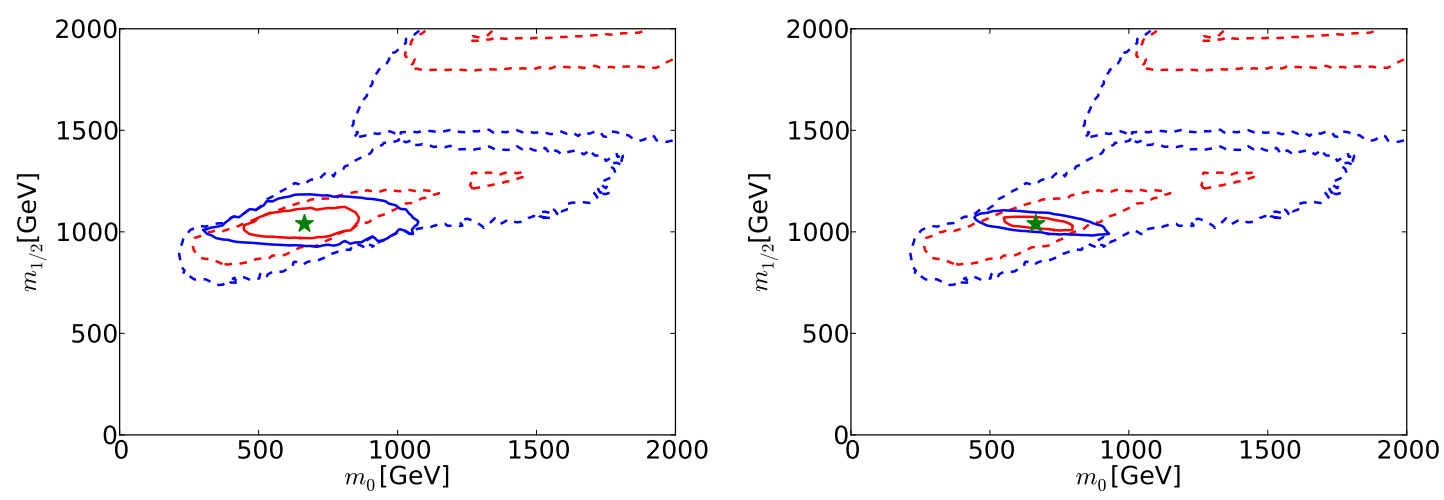

Figure 10: The solid lines show the prospective results of fits combining LHC measurements of the crosssection, MT2 and supplementary jets at LHC14 with 300/fb (left panel) and 3000/fb (right panel) with the results of a recent global fit to the CMSSM (dashed lines): the red and blue contours represent the 68 and 95\% CL regions, respectively [29].

\section{Prospects for Discovering Supersymmetry at Future Colliders}

What if supersymmetry does not turn up at the LHC? What are the prospects for discovering it at some future collider? The constraints from the LHC already disfavour, but do not exclude, the possibility of discovering supersymmetry at a future $e^{+} e^{-}$collider. There is not much scope for discovering it at the ILC with its centre-of-mass energy $\leq 500$ or $1000 \mathrm{GeV}$, and the same is true $a$ fortiori for the CEPC or FCC-ee with their more limited centre-of-mass energies. These machines would probably be limited to indirect probes of supersymmetry, at which FCC-ee would excel [29]. However, CLIC with its higher centre-of-mass energy $\leq 3000 \mathrm{GeV}$ may have brighter prospects: important guidance will be provided by Run 2 of the LHC.

Proton-proton colliders with centre-of-mass energies higher than the LHC are attracting increased attention, notably the SppC project in China [30] and the FCC-hh project at CERN [31]. The latter would be located in a circular tunnel $\sim 80$ to $100 \mathrm{~km}$ in circumference, and aims at a centre-of-mass energy of $100 \mathrm{TeV}$. Fig. 11 displays the reaches of various $p p$ colliders for searches for squarks and gluinos [32]. With 3000/fb of integrated luminosity, a $100 \mathrm{TeV} p p$ collider would have a discovery reach $\sim 15 \mathrm{TeV}$ for the squark or gluino mass, within simplified models. Increasing the target luminosity to $20,000 / \mathrm{fb}$ is currently under discussion [33], which would increase the discovery reach to $\sim 20 \mathrm{TeV}$.

How large might the squark and masses be? Of course, fine-tuning arguments would have favoured masses $\lesssim 1 \mathrm{TeV}$, but these arguments are notoriously imprecise and subjective. The DM density provides more secure upper limits on sparticle masses in supersymmetric models, at the price of assuming $R$ parity conservation and conventional adiabatic expansion of the Universe. As discussed previously, LSP masses much above a TeV are possible only under exceptional conditions on the sparticle spectrum, such as near-degeneracy between the LSP and the NLSP so that coannihilation is possible, or when the LSP mass is close to half that of a direct-channel heavy Higgs resonance, so that LSP-LSP annihilation is enhanced.

If the LSP is almost degenerate with the lighter stop, which is possible even in the CMSSM, 


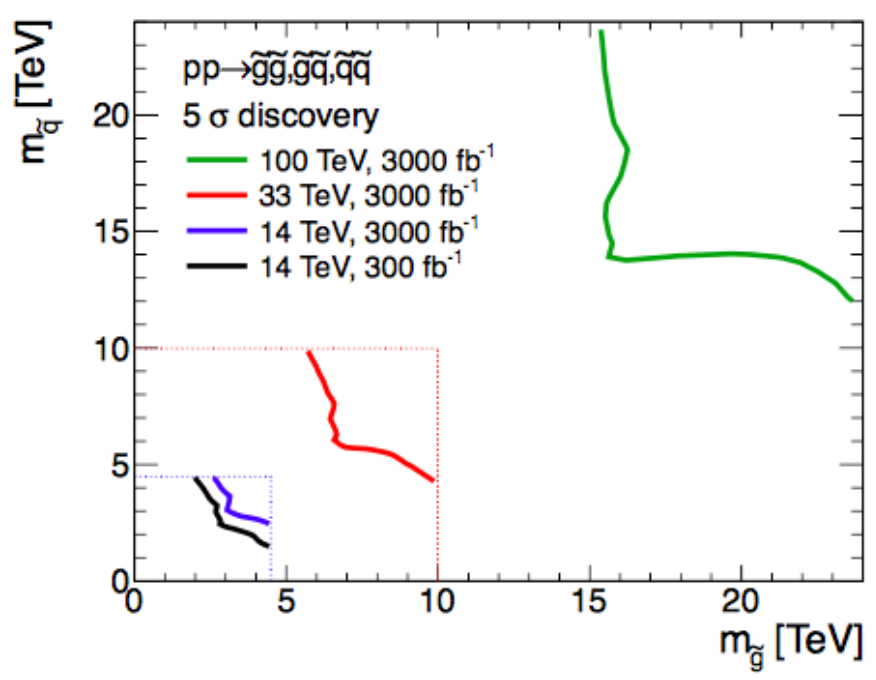

Figure 11: Reaches for discovery of the gluino, $\tilde{g}$, and a generic squark, $\tilde{q}$, at the LHC operating at $14 \mathrm{TeV}$ with 300 or 3000/fb of integrated luminosity, at a 33-TeV HE-LHC with 3000/fb, and at a 100-TeV collider with 3000/fb [32].

the LSP mass may be as large as $\sim 6.5 \mathrm{TeV}$ [34]. This would seem to be within reach of a 100$\mathrm{TeV}$ collider. However, close to the end-point of the stop coannihilation strip, the mass difference $m_{\tilde{t}_{1}}-m_{\chi} \rightarrow 0$, as seen in the left panel of Fig. 12. The black, blue, green, purple and red lines are particle exclusion reaches for particle searches with LHC at $8 \mathrm{TeV}, 300$ and 3000/fb with LHC at 14 $\mathrm{TeV}, 3000 / \mathrm{fb}$ with HE-LHC at $33 \mathrm{TeV}$ and 3000/fb with FCC-hh at $100 \mathrm{TeV}$, respectively. The solid lines are for generic MET searches, and the dashed lines are for dedicated stop searches. Towards the end of the stop coannihilation strip, stop decays would not produce energetic jets and might be difficult to detect directly. Nevertheless, in the CMSSM there would be other supersymmetric signatures due, e.g., to the production and decays of gluinos and other squark flavours. Thus the CMSSM stop coannihilation scenario should be accessible at a 100-TeV pp collider, as shown by the solid red line in the left panel of Fig. 12.

The LSP could be even heavier, $m_{\chi} \lesssim 8 \mathrm{TeV}$, if it coannhilates with the gluino [35]. This is not possible in the CMSSM, but is possible in models with non-universal gaugino masses: $M_{1} \neq M_{3}$ at the input GUT scale, or in pure gravity mediation models with extra vector-like supermultiplets [36]. An example of the former is shown in the right panel of Fig. 12. In this case the direct production of gluino pairs would produce energetic jets towards the tip of the gluino coannihilation strip, and the input scalar mass $m_{0}=20 \mathrm{TeV}$, rendering squarks difficult to detect. Moreover, larger values of $m_{0}$ are also possible. Therefore the tip of the coannihilation strip where $m_{\chi} \sim m_{\tilde{g}} \sim 8 \mathrm{TeV}$ could be challenging even for a 100-TeV $p p$ collider, though associated gluino + squark production offers some prospects if $m_{0}$ is not too large [37].

\section{Summary}

Rumours of the death of supersymmetry are greatly exaggerated. I consider that it is still the best-motivated framework for $\mathrm{TeV}$-scale physics, in view of its help in making the fine-tuning of the 

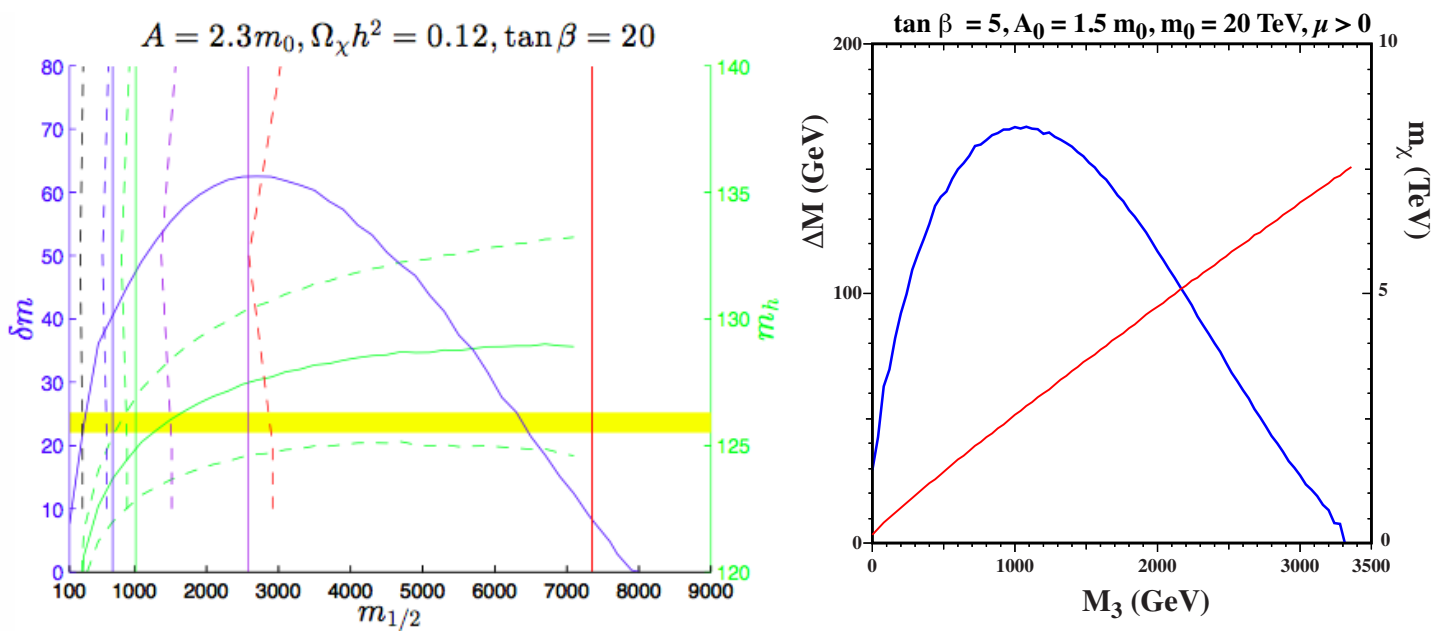

Figure 12: Profiles of coannihilation strips corresponding to $\Omega_{\chi} h^{2}=0.120$ for (left panel) the lighter stop, $\tilde{t}_{1}$, in the CMSSM for $A_{0}=2.3 m_{0}$ and $\tan \beta=20$ [34], and (right panel) the gluino, $\tilde{g}$, in a variant of the CMSSM with non-universal gaugino masses $M_{!}=M_{2} \neq M_{3}, m_{0}=20 \mathrm{TeV}, A_{0}=1.5 m_{0}$ and $\tan \beta=20$ [36], both with $\mu>0$.

mass hierarchy more natural, its help with grand unification and its rôle in string theory. In addition, the LSP is still an excellent candidate for cold dark matter. All that said, simple supersymmetric models with universal soft supersymmetry-breaking terms, such as the CMSSM, etc., are under pressure. However, it is worth noting that much of the pressure comes from $g_{\mu}-2$, which is even more of an issue for the SM. Moreover, more general models within the pMSSM framework are quite healthy, since they can reconcile $g_{\mu}-2$ with the other constraints. Within the CMSSM, the NUHM1, the NUHM2 and the pMSSM10 we find good prospects for detecting supersymmetry and possibly making some interesting measurements, either at LHC Run 2 or via direct dark matter detection, though there are no guarantees. We may well need a higher-energy $p p$ collider, either to make follow-up studies of supersymmetry, or to discover it!

\section{References}

[1] D. Buttazzo, G. Degrassi, P. P. Giardino, G. F. Giudice, F. Sala, A. Salvio and A. Strumia, JHEP 1312 (2013) 089 [arXiv:1307.3536 [hep-ph]].

[2] ATLAS, CDF, CMS and D0 Collaborations, arXiv:1403.4427 [hep-ex].

[3] G. Aad et al. [ATLAS and CMS Collaborations], Phys. Rev. Lett. 114 (2015) 191803 [arXiv:1503.07589 [hep-ex]].

[4] K.A. Olive et al. (Particle Data Group), Chin. Phys. C, 38, 090001 (2014).

[5] V. Khachatryan et al. [CMS Collaboration], arXiv:1509.04044 [hep-ex].

[6] S. Bethke, Workshop on high-precision $\alpha_{s}$ measurements: from LHC to FCC-ee, https://indico.cern.ch/event/392530/contribution/1/attachments/ 1168353/1686119/alphas-CERN2015.pdf.

[7] See, e.g., M. Fairbairn and R. Hogan, Phys. Rev. Lett. 112 (2014) 201801 [arXiv:1403.6786 [hep-ph]]; A. Hook, J. Kearney, B. Shakya and K. M. Zurek, JHEP 1501 (2015) 061 [arXiv:1404.5953 [hep-ph]]. 
[8] J. R. Ellis and D. Ross, Phys. Lett. B 506 (2001) 331 [hep-ph/0012067].

[9] See, e.g., V. Branchina, E. Messina and M. Sher, Phys. Rev. D 91 (2015) 013003 [arXiv:1408.5302 [hep-ph]]. However, see also L. Di Luzio, G. Isidori and G. Ridolfi, arXiv:1509.05028 [hep-ph].

[10] J. R. Ellis, G. Ridolfi and F. Zwirner, Phys. Lett. B 257 (1991) 83; H. E. Haber and R. Hempfling, Phys. Rev. Lett. 66 (1991) 1815; Y. Okada, M. Yamaguchi and T. Yanagida, Prog. Theor. Phys. 85 (1991) 1 .

[11] J. R. Ellis, S. Heinemeyer, K. A. Olive and G. Weiglein, JHEP 0301 (2003) 006 [hep-ph/0211206].

[12] H. Goldberg, Phys. Rev. Lett. 50 (1983) 1419 [Phys. Rev. Lett. 103 (2009) 099905]; J. R. Ellis, J. S. Hagelin, D. V. Nanopoulos, K. A. Olive and M. Srednicki, Nucl. Phys. B 238 (1984) 453.

[13] M. McCullough, talk at the 2015 Lepton-Photon Symposium, https : / / indico. cern. ch/ event/325831/session/6/contribution/13/attachments/1140468/ 1633470 /Lept onPhot on .pdf; Z. Chacko, H. S. Goh and R. Harnik, Phys. Rev. Lett. 96 (2006) 231802 [hep-ph/0506256]; P. W. Graham, D. E. Kaplan and S. Rajendran, arXiv:1504.07551 [hep-ph]. See also G. Dvali and A. Vilenkin, Phys. Rev. D 70 (2004) 063501 [hep-th/0304043] and G. Dvali, Phys. Rev. D 74 (2006) 025018 [hep-th/0410286].

[14] O. Buchmueller et al., Eur. Phys. J. C 74 (2014) 6, 2922 [arXiv:1312.5250 [hep-ph]].

[15] O. Buchmueller et al., Eur. Phys. J. C 74 (2014) 12, 3212 [arXiv:1408.4060 [hep-ph]].

[16] K. J. de Vries et al., Eur. Phys. J. C 75 (2015) 9, 422 [arXiv:1504.03260 [hep-ph]].

[17] For an alternative frequentist analysis of the CMSSM, see P. Bechtle et al., arXiv:1508.05951 [hep-ph].

[18] For Bayesian analyses of the CMSSM and NUHM, see L. Roszkowski, E. M. Sessolo and A. J. Williams, JHEP 1408 (2014) 067 [arXiv:1405.4289 [hep-ph]] and references therein.

[19] E. A. Bagnaschi et al., arXiv:1508.01173 [hep-ph].

[20] K. de Vries, PhD thesis, 2015.

[21] G. W. Bennett et al. [Muon g-2 Collaboration], Phys. Rev. D 73 (2006) 072003 [hep-ex/0602035].

[22] V. Khachatryan et al. [CMS and LHCb Collaborations], Nature 522 (2015) 68 [arXiv:1411.4413 [hep-ex]].

[23] M. Citron, J. Ellis, F. Luo, J. Marrouche, K. A. Olive and K. J. de Vries, Phys. Rev. D 87 (2013) 3, 036012 [arXiv:1212.2886 [hep-ph]].

[24] N. Desai, J. Ellis, F. Luo and J. Marrouche, Phys. Rev. D 90 (2014) 5, 055031 [arXiv:1404.5061 [hep-ph]].

[25] M. Papucci, K. Sakurai, A. Weiler and L. Zeune, Eur. Phys. J. C 74 (2014) 11, 3163 [arXiv:1402.0492 [hep-ph]] and Atom: Automated Tests of Models, in preparation.

[26] Scorpion was first developed by J. Marrouche, and developed further by O. Buchmueller, M. Citron, S. Malik and K.J. de Vries: details may be obtained by contacting O. Buchmueller.

[27] P. Bechtle, S. Heinemeyer, O. StŇl, T. Stefaniak and G. Weiglein, Eur. Phys. J. C 74 (2014) 2, 2711 [arXiv:1305.1933 [hep-ph]]; JHEP 1411 (2014) 039 [arXiv:1403.1582 [hep-ph]].

[28] Fermilab Muon $g-2$ Experiment: http://muon-g-2. fnal.gov. 
[29] O. Buchmueller, M. Citron, J. Ellis, S. Guha, J. Marrouche, K. A. Olive, K. de Vries and J. Zheng, Eur. Phys. J. C 75 (2015) 10, 469 [arXiv:1505.04702 [hep-ph]].

[30] CEPC-SppC Study Group: CEPC pre-CDR, http://cepc.ihep.ac.cn/preCDR/volume.html.

[31] Future Circular Collider Study, https: / / fcc.web. cern. ch/Pages/default.aspx.

[32] T. Cohen, T. Golling, M. Hance, A. Henrichs, K. Howe, J. Loyal, S. Padhi and J. G. Wacker, JHEP 1404 (2014) 117 [arXiv:1311.6480 [hep-ph]].

[33] I. Hinchliffe, A. Kotwal, M. L. Mangano, C. Quigg and L. T. Wang, Int. J. Mod. Phys. A 30 (2015) 1544002 [arXiv:1504.06108 [hep-ph]].

[34] J. Ellis, K. A. Olive and J. Zheng, Eur. Phys. J. C 74 (2014) 2947 [arXiv:1404.5571 [hep-ph]].

[35] J. Ellis, F. Luo and K. A. Olive, JHEP 1509 (2015) 127 [arXiv:1503.07142 [hep-ph]].

[36] J. Ellis, J. L. Evans, F. Luo and K. A. Olive, arXiv:1510.03498 [hep-ph].

[37] S. A. R. Ellis and B. Zheng, Phys. Rev. D 92 (2015) 7, 075034 [arXiv:1506.02644 [hep-ph]]. 